\title{
Qualitative Histopathologic Examination
}

National Cancer Institute

\section{Source}

National Cancer Institute. Qualitative Histopathologic Examination. NCI Thesaurus. Code C147493.

A general histopathologic examination, without a prespecified visual target, focused on qualitative or semi-quantitative findings. 